\title{
The MoEDAL Experiment at the LHC - Searching for Physics Beyond the Standard Model
}

\author{
James Pinfold ${ }^{1, a}$ For the MoEDAL Collaboration \\ ${ }^{1}$ University of Alberta
}

\begin{abstract}
MoEDAL is a pioneering experiment designed to search for highly ionizing messengers of new physics such as magnetic monopoles or massive (pseudo-)stable charged particles, that are predicted to exist in a plethora of models beyond the Standard Model. It started data taking at the LHC at a centre-of-mass energy of $13 \mathrm{TeV}$, in 2015. MoEDAL's ground breaking physics program defines a number of scenarios that yield potentially revolutionary insights into such foundational questions as: are there extra dimensions or new symmetries; what is the mechanism for the generation of mass; does magnetic charge exist; and what is the nature of dark matter. MoEDAL's purpose is to meet such far-reaching challenges at the frontier of the field. We will present an overview of the MoEDAL detector, including the planned MAPP subdetector, as well as MoEDAL's physics program. The concluding section highlights our first physics results on Magnetic Monopole production, that are the world's best for Monopoles with multiple magnetic charge.
\end{abstract}

\section{Introduction}

The MoEDAL experiment, the newest LHC experiment, started data taking in 2015. MoEDAL is a pioneering experiment designed to search for highly ionizing avatars of new physics such as magnetic monopoles or massive (pseudo-)stable charged particles. It is largely deployed in the LHCb's VELO cavern at Point 8 (IP8) on the LHC ring. Its groundbreaking physics program defines over 30 scenarios that yield potentially revolutionary insights into such foundational questions as: are there extra dimensions or new symmetries; what is the mechanism for the generation of mass; does magnetic charge exist; what is the nature of dark matter; and, how did the big-bang develop. MoEDAL's purpose is to meet such far-reaching challenges at the frontier of the field.

The innovative MoEDAL detector employs unconventional methodologies tuned to the prospect of discovery physics. The largely passive MoEDAL detector has a dual nature. First, it acts like a giant camera, comprised of nuclear track detectors - analyzed offline by ultra fast scanning microscopes sensitive only to new physics. Second, it is uniquely able to trap the particle messengers of physics beyond the Standard Model for further study. MoEDAL's radiation environment is monitored by a state-of-the-art real-time TimePix pixel detector array. MoEDAL is currently installing a prototype of the MAPP (MoEDAL Apparatus for very Penetrating Particles) sub-detector, in a tunnel in the vicinity of LHCb/MoEDAL at IP8. This new subdetector is designed to search for very long-lived highly penetrating particles such a mini-charged particles, long lived neutrals, etc.

ae-mail: \pinfold@ualberta.ca 


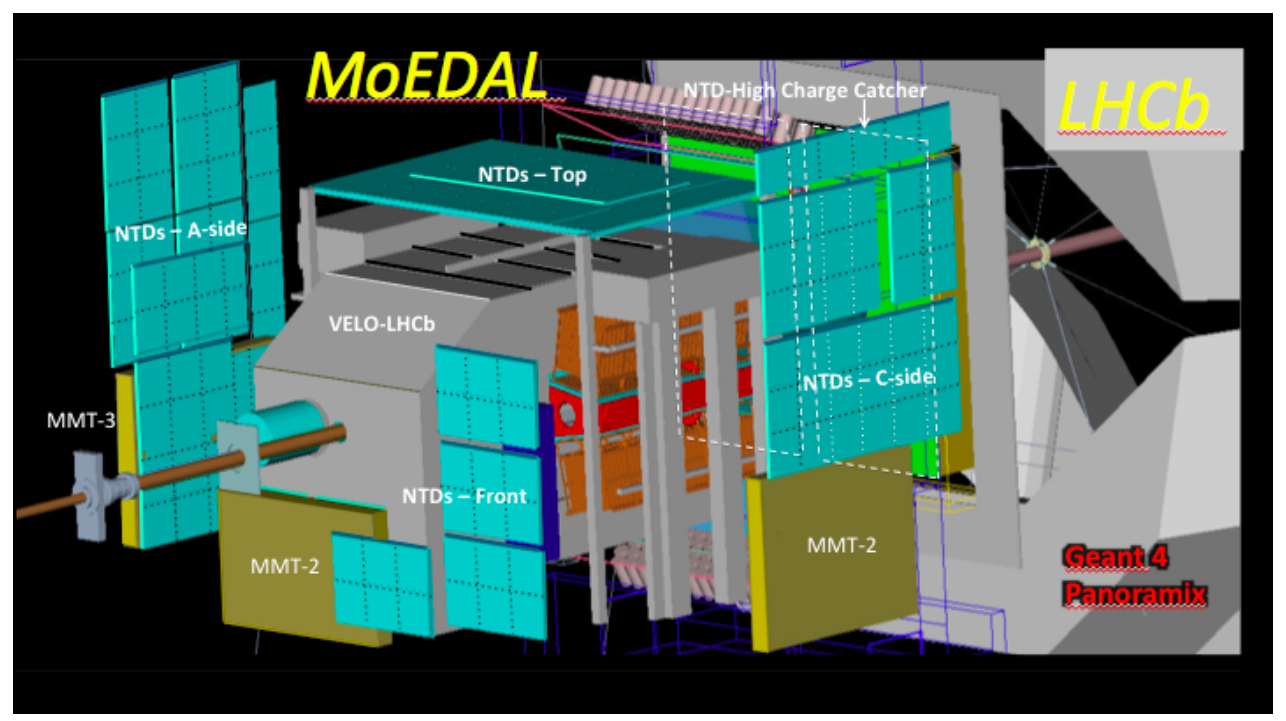

Figure 1. A GEANT-4 "Panoramix" depiction of the baseline MoEDAL detector.

\section{The MoEDAL Detector}

MoEDAL is an unconventional and largely passive LHC detector comprised of the largest array of plastic (CR39 and Makrofol) Nuclear Track Detector stacks ever deployed at an accelerator, surrounding the intersection region at Point 8 on the LHC ring. Another novel feature is the use of paramagnetic (aluminium) trapping volumes to capture both electrically and magnetically charged highly-ionizing particles predicted in new physics scenarios. It includes an array of TimePix pixel devices for monitoring highly-ionizing particle backgrounds. The main passive elements of the MoEDAL detector do not require a trigger system, electronic readout, or online computerized data acquisition. A GEANT-4 "Panoramix" depiction of the MoEDAL detector is given in Figure 1.

MoEDAL's planned MAPP subdetector is based on conventional scintillator detector technology readout by PMTs at each end of each scintillator bar. MAPP takes advantage of its location - in the UGC8 gallery - where it is protected from Standard Model background originating from collisions at IP8 by $30 \mathrm{~m}$ to $50 \mathrm{~m}$ or rock. A schematic depiction of the location of MAPP is shown in Figure 2. The baseline MoEDAL detector and its MAPP extension is designed to be largely complementary in its sensitivity to the main general-purpose LHC experiments ATLAS and CMS.

In order to study the background conditions a prototype MAPP detector will be deployed at IP8 in the Winter of 2017/2018. A photograph of the detectors (without PMTs attached) is shown in Figure 3. The length of the prototype is $2 \mathrm{~m}$, with PMTs attached. It is likely that the final detector will be subdivided into 2 separate longitudinal sections. Each end of each bar in both sections will be readout by PMTs. For a track to be registered a coincidence between each pair of PMTs in a collinear set of scintillator bars, must be obtained. 


\section{MAPP (MoEDAL Apparatus for detection of Penetrating Particles)}

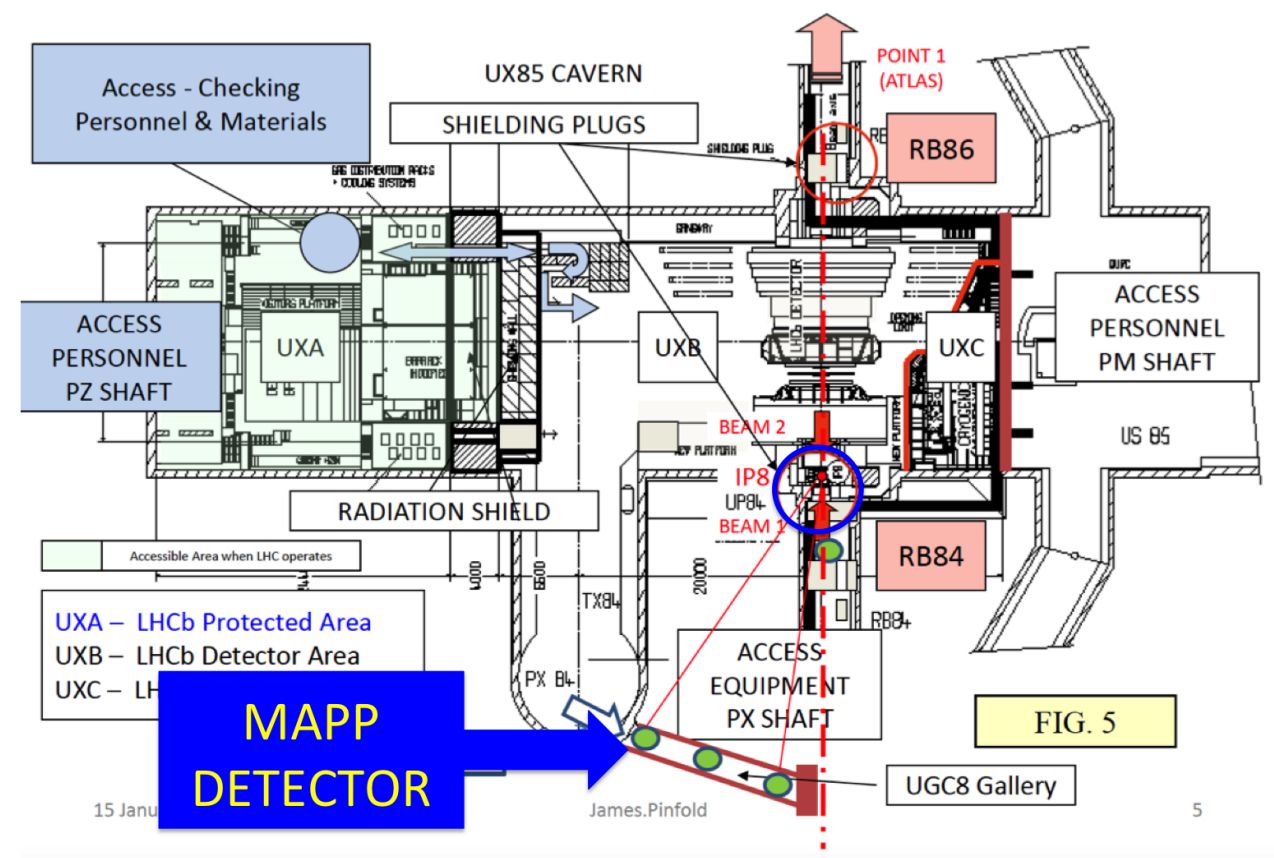

Figure 2. The location of the MAPP subdetector

\section{The Physics Program}

The most important motivation for the MoEDAL experiment is to continue the quest for the magnetic monopole [1] to LHC energies. However, the experiment is also designed to search for massive, stable or long-lived, slow-moving particles [2] with single or multiple electric charges that arise in many scenarios of physics beyond the Standard Model (BSM) [1]. An overview of the over 30 physics topics that can be explored using the baseline experiment is shown in Figure 4. The MAPP sub-detector will give access to a number of additional new physics scenarios involving fractionally charged particles and long lived neutral particles, etc. The MoEDAL physics reach is largely complementary to the that of the large multi-purpose LHC detectors ATLAS and CMS.

Apart from magnetically charged particles, MoEDAL is also searching for (meta)stable electrically-charged massive particles. Such particles arise in numerous scenarios (see Figure 4) involving, for example, extra dimensions and new symmetries of nature, such as supersymmetry. MoEDAL can extend significantly the discovery reach of the LHC with respect to such states thanks to its trigger-free concept of passive detectors, no necessity for strong kinematic requirements for signal detection, and, no requirement for high thresholds to handle high luminosity running. Although, there is a requirement for the ratio of the charge to the velocity $(\mathrm{Z} / \beta)$ to exceed the threshold for detection in the plastic. Another limiting factor is the comparatively low luminosity delivered at IP8. However, this llimitation will be ameliorated after the upcoming LHC shutdown at which time the luminosity available at point 8 will have been increased by a factor of $\sim 5$. 


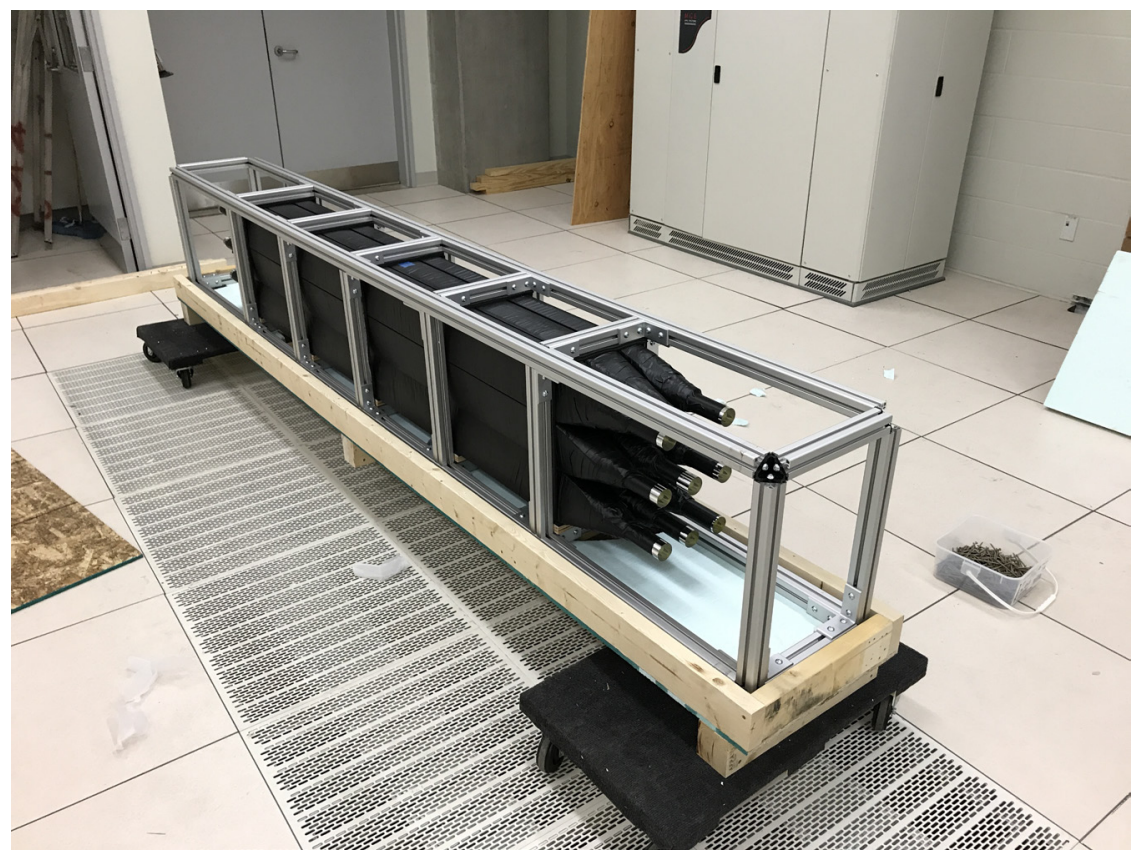

Figure 3. A photograph of the MAPP prototype subdetector without PMTs attached

A good example of the ability to MoEDAL to extend the LHC sensitivity to, say, signals of supersymmetric particle production is gluino pair production $(\tilde{g} \tilde{g})$, where $\tilde{g} \rightarrow j j \tilde{\chi}_{1}^{0}$ and $\tilde{\chi} \rightarrow \tau^{ \pm} \tilde{\tau}_{1}$. In this case, the $\tilde{\chi}_{1}^{0}$ is long lived despite the large mass split between $\tilde{\chi}_{1}^{0}$ and $\tilde{\tau}_{1}$ decays in the tracker. The decay of the $\tau$ produces a kink between the $\tilde{\chi}_{1}^{0}$ and $\tilde{\tau}_{1}$ tracks. The tracks resulting from the $\tau$ decay have a large impact parameter with the event vertex. Also, in this scenario, the $\tilde{\tau}_{1}$ is metastable, as is the case with a gravitino LSP. The comparison of the CMS exclusion with the MoEDAL discovery potential, requiring just one (left plot) or three(right plot) events, is shown in Figure 5. This shows that MoEDAL can cover the long-lifetime region inaccessible to ATLAS/CMS even with a high NTD threshold of $Z / \beta$ of 10. A more detailed discussion of MoEDAL's potential in the search for supersymmetry can be found elsewhere [3].

\section{MoEDAL Results and Current Status}

MoEDAL first published physics analysis reported a search for magnetic monopoles using a $160 \mathrm{~kg}$ prototype MoEDAL trapping detector exposed to $8 \mathrm{TeV}$ proton-proton collisions [4] at the LHC, for an integrated luminosity of $0.75 \mathrm{fb}^{-1}$. In this case monopoles were sought by passing the trapping volumes through a superconducting magnetometer (SQUID) in order to detect the presence of induced persistent currents caused by the passage of a monopole through the SQUID. No magnetic charge exceeding $0.5 \mathrm{~g}_{D}$ (where $\mathrm{g}_{D}$ is the Dirac magnetic charge) was measured in any of the exposed samples, allowing limits to be placed on monopole production in the mass range $100 \mathrm{GeV} \leq m \leq 3500 \mathrm{GeV}$. Model-independent cross-section limits are presented in fiducial regions of monopole energy and direction for $1 \mathrm{~g}_{D} \leq|g| \leq 6 \mathrm{~g}_{D}$, and model-dependent cross-section limits are obtained for Drell-Yan 


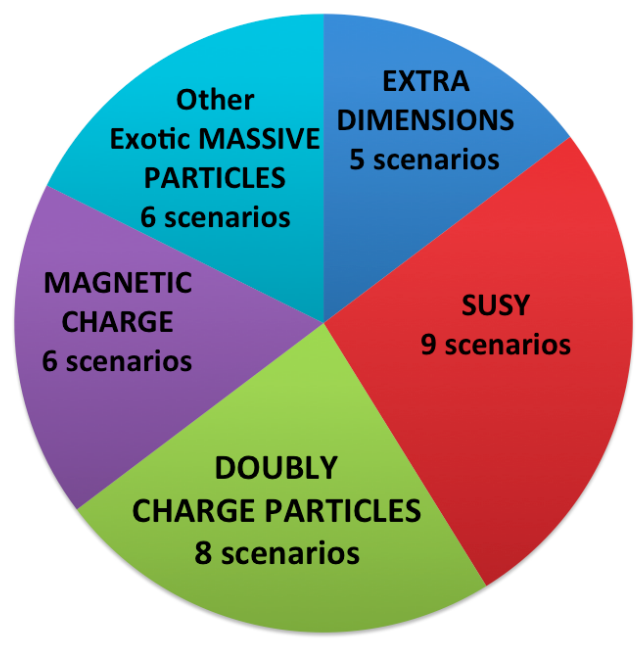

Figure 4. A pie chart describing the topics covered by MoEDAL's physics program
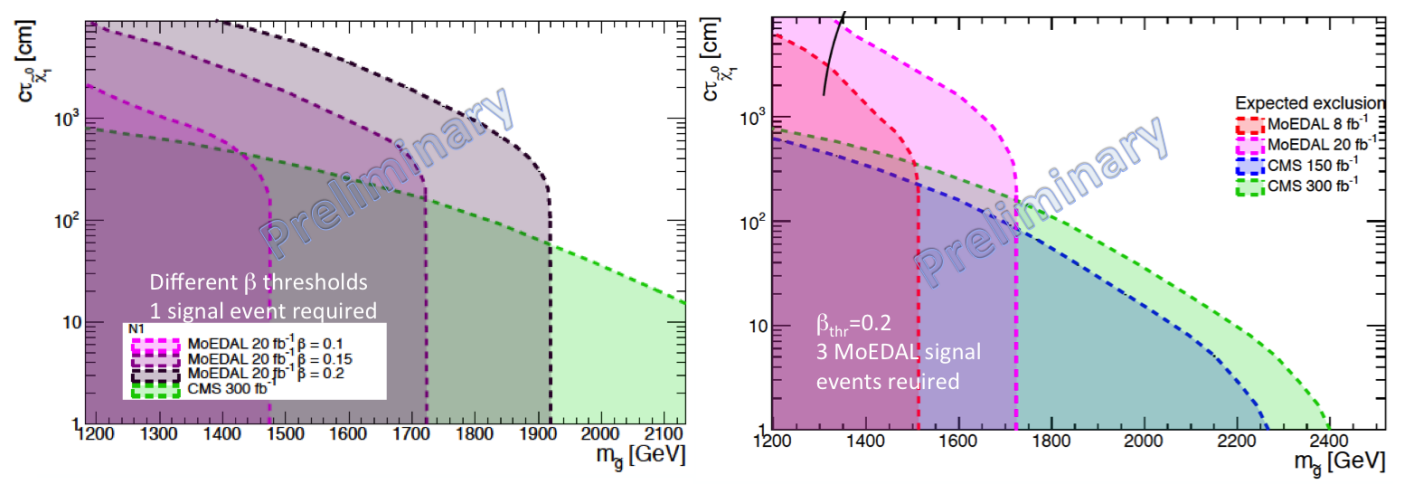

Figure 5. Comparison of CMS exclusion with MoEDAL discovery potential requiring 1 candidate event (left plot) and 3 candidate events (right plot), per channel.

pair production of spin-1/2 and spin-0 monopoles for $1 \mathrm{~g}_{D} \leq|g| \leq 4 \mathrm{~g}_{D}$. Under the assumption of Drell-Yan cross sections, mass limits are derived for $|\mathrm{g}|=2 \mathrm{~g}_{D}$ and $|\mathrm{g}|=3 \mathrm{~g}_{D}$ for the first time at the LHC, surpassing the results from previous collider experiments.

A second MoEDAL analysis presented the search for magnetic monopole production in $13 \mathrm{TeV}$ (Run-2) proton-proton collisions [5] using the trapping technique, extending our previous publication with $8 \mathrm{TeV}$ data during LHC run-1. In this case, a total of $222 \mathrm{~kg}$ of MoEDAL trapping detector samples was exposed in the forward region and then monitored for the presence of magnetic monopoles in a data sample corresponding to a luminosity of $0.371 \pm 0.004 \mathrm{fb}^{-1}$ obtained during the 2015 exposure to $13 \mathrm{TeV}$ pp collisions. The results of both analyses are summarized in Figure 6 and 


\begin{tabular}{|l|c|c|c|c|}
\hline mass limits [GeV] & $1 g_{\mathrm{D}}$ & $2 g_{\mathrm{D}}$ & $3 g_{\mathrm{D}}$ & $4 g_{\mathrm{D}}$ \\
\hline MoEDAL 13 TeV & & & & \\
(this result) & & & & \\
DY spin-1/2 & 890 & 1250 & 1260 & 1100 \\
DY spin-0 & 460 & 760 & 800 & 650 \\
\hline MoEDAL 8 TeV & & & & \\
DY spin-1/2 & 700 & 920 & 840 & - \\
DY spin-0 & 420 & 600 & 560 & - \\
\hline ATLAS 8 TeV & & & & \\
DY spin-1/2 & 1340 & - & - & - \\
DY spin-0 & 1050 & - & - & - \\
\hline
\end{tabular}

Figure 6. Monopole lower mass limits (95\% confidence level) in models of spin-1/2 and spin-0 DY pair production in LHC pp collisions for monopole charges $|\mathrm{g}|$ up to $4 \mathrm{~g}_{D}$. These limits are based upon cross sections computed at leading order. These cross sections are only indicative since the monopole coupling to the photon is too large to allow for perturbative calculations. Previous results obtained in $8 \mathrm{TeV}$ collisions are from Ref. [4] (MoEDAL prototype trapping detector) and Ref. [6] (ATLAS).
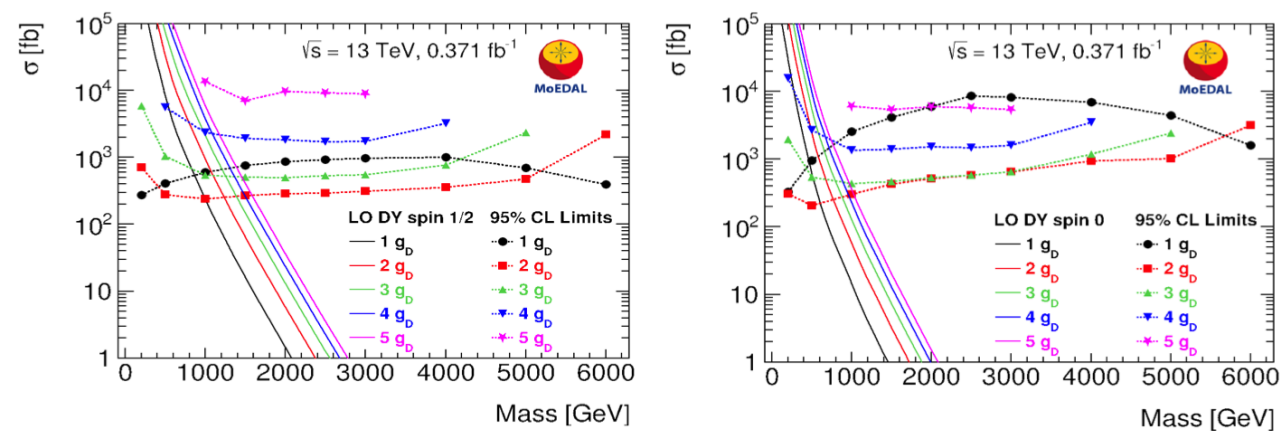

Figure 7. Cross-section upper limits at $95 \%$ confidence level for DY monopole production in $13 \mathrm{TeV}$ pp collisions as a function of mass for spin-1/2 (left) and spin-0 (right) monopoles. The colours correspond to different monopole charges. Acceptance loss is dominated by monopoles punching through the trapping volume for $|\mathrm{g}|=$ $\mathrm{g}_{D}$ while it is dominated by stopping in upstream material for higher charges, explaining the shape difference. The solid lines are DY cross-section calculations at leading order.

Figure 7. Monopole-pair direct production cross-section limits in the range $200 \rightarrow 10000 \mathrm{fb}$ were set for magnetic charges up to $5 \mathrm{~g}_{D}$ and masses up to $6 \mathrm{TeV}$. In a DY model with spin-1/2 monopoles, this translates into monopole mass limits exceeding $1 \mathrm{TeV}$ for charges ranging from two to four times the Dirac charge - the strongest to date at a collider experiment. These were the first monopole search results published at $13 \mathrm{TeV}$ LHC centre-of-mass energy. 
Currently, we have several analyses in the pipeline where the full functionality of the MoEDAL detector is employed to search for anomalously ionizing new physics particles in both proton-proton and heavy ion collisions.

\section{References}

[1] For example: B. Acharya et al., Int. J. Mod. Phys. A29, 1430050 (2014).

[2] For example: M. Fairbairn, A. C. Kraan, D. A. Milstead, T. Sjostrand, P. Z. Skands and T. Sloan, Phys. Rept. 438, 1 (2007).

[3] See paper by Vasiliki Mitsou in these proceedings.

[4] B. Acharya et al., JHEP 1608, 067 (2016).

[5] B. Acharya et al., Phys. Rev. Lett. 118,no. 6, 061801 (2017).

[6] G. Aad et al, ATLAS Collaboration, Phys. Rev. D93, 052009 (2016). 